\title{
Negative ion sound solitary waves revisited
}

\author{
R. A. CAIR N S \\ School of Mathematics and Statistics, University of St Andrews, \\ St Andrews KY16 9SS, UK \\ (rac@st-andrews.ac.uk)
}

(Received 12 August 2013; revised 12 August 2013; accepted 14 August 2013; first published online 4 November 2013)

\begin{abstract}
Some years ago, a group including the present author and Padma Shukla showed that a suitable non-thermal electron distribution allows the formation of ion sound solitary waves with either positive or negative density perturbations, whereas with Maxwellian electrons only a positive density perturbation is possible. The present paper discusses the qualitative features of this distribution allowing the negative waves and shared with suitable two-temperature distributions.
\end{abstract}

\section{Introduction}

Some time ago, in a paper by the present author, Padma Shukla and others (Cairns et al. 1995), the possibility of having ion sound waves with either positive or negative density perturbations was demonstrated. The work was motivated by spacecraft observations of structures in the ionosphere involving density depressions and showed that a non-thermal distribution of electrons could allow formation of an ion sound solitary wave with such a density depression, in contrast to the situation with a Maxwellian distribution when only solitary waves with an increased density are possible. The distribution we proposed was an ad hoc construction, designed to mimic the effect of higher frequency turbulence in flattening the electron distribution function, but has since been adopted by many authors as a model for non-thermal effects. It has been referred to as the 'Cairns distribution' by a number of authors so, for convenience, this nomenclature is adopted here. The question addressed is just what property of the Cairns distribution leads to the existence of both positive and negative density ion sound solitary waves, a property which it does not appear to share with other non-thermal distributions such as the kappa or Tsallis distributions (Chuang and Lau 2009). It is, however, possible to have negative solitary waves with two-temperature Maxwellian or kappa distributions, detailed discussions having been given by Baluku and co-workers (Baluku et al. 2010; Baluku and Hellberg 2012). It will be shown that the behaviour of the high-energy tail of the distribution is not the crucial feature but that the feature of the Cairns distribution which allows negative waves is the flattening of the distribution at lower velocities.

\section{Theory}

The Cairns distribution for the electrons takes the form, in one dimension,

$$
f(v)=\frac{n_{0}}{\sqrt{2 \pi V_{e}^{2}}} \frac{1}{3 \alpha+1}\left(1+\alpha \frac{v^{4}}{V_{e}^{4}}\right) \exp \left(-\frac{v^{2}}{2 V_{e}^{2}}\right),
$$

with $n_{0}$ the equilibrium density, $V_{e}$ a characteristic thermal velocity (though note that the energy density is not $\frac{1}{2} m_{e} V_{e}^{2}$ if $\alpha$ is non-zero), and $\alpha$ a constant which determines the deviation from Maxwellian of the distribution. Note that $\alpha$ cannot be too large, otherwise the distribution takes on an unstable bump on tail form. In our previous work, we chose a value 0.2 and will use the same value here for any numerical illustrations. In the presence of a non-zero electric potential $\phi$, we made the usual assumption that the distribution takes the form of (1) with $v^{2}$ replaced by $v^{2}-\frac{2 e \phi}{m_{e}}$, with $e$ and $m_{e}$ be the electron charge and mass. If we denote the resulting function by $n_{0} F(u)$ with $u=v^{2}-\frac{2 e \phi}{m_{e}}$, then the electron density is

$$
n_{e}=n_{0} \int_{-\infty}^{\infty} F(u) d v
$$

The ions, of mass $m_{i}$, are taken to be cold so that, in a frame of reference moving with the speed $V$ of the solitary structure, the ion density is

$$
n_{i}=\frac{n_{0} V}{\sqrt{V^{2}-\frac{2 e \phi}{m_{i}}}},
$$

where we have assumed singly charged ions for convenience. Poisson's equation gives

$$
\frac{d^{2} \phi}{d x^{2}}=-\frac{e}{\varepsilon_{0}}\left(n_{i}-n_{e}\right)=-\frac{d \Phi}{d \phi},
$$

where $\Phi(\phi)$ is the Sagdeev potential (Sagdeev 1966), introduced so that (4) is precisely analogous to the equation of motion of a particle in the potential $\Phi$. To obtain a solitary wave with a density depression requires that $\phi$ be negative within the wave. For such a solution to exist we need $\Phi^{\prime \prime}(0)<0$, so that the origin is an unstable point and for there to be some negative 
value of $\phi$ at which $\Phi=0$ (taking $\Phi(0)=0$ ). Under these conditions there is a homoclinic orbit in the phase space of (4), starting and finishing at the origin and producing the required solitary wave.

Now let us consider a more general electron distribution, still a function of $v^{2}-\frac{2 e \phi}{m_{e}}$, so that we have (2) but with $F$, a more general function, normalized so that its integral over velocity is one. The condition that $\Phi^{\prime \prime}(0)<0$ becomes

$$
\frac{n_{0} e}{m_{i} V^{2}}+\frac{2 n_{0} e}{m_{e}} \int_{-\infty}^{\infty} F^{\prime}\left(v^{2}\right) d v<0 .
$$

The integral in the second term here has the dimensions of velocity ${ }^{-2}$, so we denote it by $2 v_{0}^{-2}$, making the condition (5) $m_{i} V^{2}>m_{e} v_{0}^{2}$. For a Maxwellian $F\left(v^{2}\right)=$ $\frac{1}{\left(2 \pi V_{e}^{2}\right)} e^{-v^{2} / 2 V_{e}^{2}}$ so that (5) gives $V^{2}>\frac{m_{e} V_{e}^{2}}{m_{i}}$, the usual condition that the ion sound Mach number be greater than one. For the Cairns distribution, the condition becomes $V^{2}>\frac{3 \alpha+1}{\alpha-1} \frac{m_{e} V_{e}^{2}}{m_{i}}$, as given in Cairns et al. (1995).

Turning now to the other requirement, that $\Phi$ returns to zero at some negative value, this needs $\Phi^{\prime \prime}$ to change sign away from $\phi=0$ so that $\Phi$ curves upwards to meet the axis. From Poisson's equation, it is clear that a necessary condition for this is that the electron density exceeds the ion density for some negative value of $\phi$. For large negative values of $\phi$, the ion density goes as $|\phi|^{-1 / 2}$ while the electron density falls off more rapidly because of the exponential factor. For a kappa distribution, suggested originally by Vasyliunas as a good model for non-thermal distributions in space plasmas (Vasyliunas 1968), the distribution goes as $\left(1+\frac{v^{2}}{2 k V_{e}^{2}}\right)^{-(\kappa+1)}$. A distribution with a similar power law tail was later obtained by Tsallis (1988) using a modification of thermodynamics in which entropy is a non-extensive quantity, an assumption which may be valid for a plasma because of its long-range correlations. For the kappa distribution, the electron density goes as $|\phi|^{-(\kappa+1 / 2)}$ for large negative $\phi$. For the distribution to have finite energy requires $\kappa>1 / 2$ so again the electron distribution falls off more rapidly than the ion distribution for large negative $\phi$. A similar conclusion will hold for any distribution with finite energy. We can conclude from this that it is not the tail of the distribution and the asymptotic behaviour of $\phi$ that determines the existence of the negative soliton but rather the behaviour at smaller values of $\phi$. For sufficiently small negative values of $\phi$, the electron density is again less than the ion density because of the condition $\Phi^{\prime \prime}(0)<0$. What is needed then is for there to be some intermediate range of negative potential where the electron density falls off more slowly than the ion density. The rate of change of electron density with $\phi$ is proportional to

$$
H(\phi)=-\int_{-\infty}^{\infty} F^{\prime}\left(v^{2}-2 e \phi\right) d v
$$

For negative $\phi$, this integral involves the gradient of $F$ for values of its argument greater than $|2 e \phi|$ and

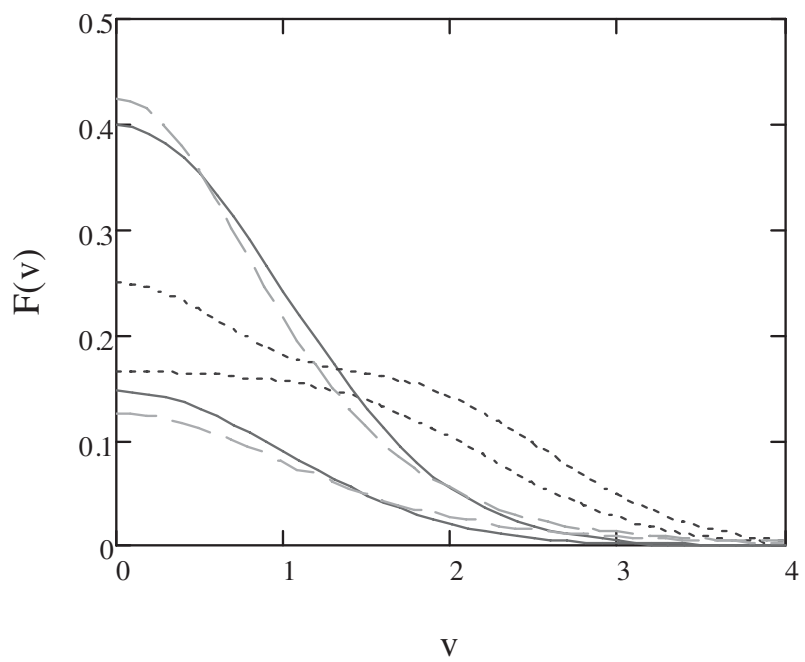

Figure 1. Maxwellian (continuous red), Kappa with $\kappa=2$ (green dashed) and Cairns (blue dotted) distributions at $\phi=0$ and $\phi=-1$. In each case, the upper curve is at $\phi=0$.

becomes small for the Cairns distribution because of its flat shoulder. Figure 1 shows the way in which Maxwellian, kappa and Cairns distributions change as $\phi$ goes from zero to -1 (normalized to $m_{e} V_{t h}^{2} / e$ ).

It is clear from this figure that the difference in electron density between the two potentials is much less for the Cairns distribution than for the other two. This illustrates the effect of the flat shoulder on the Cairns distribution. The kappa distribution, on the other hand, falls off more steeply than the Maxwellian near the origin, with the result that its density decreases more rapidly with $\phi$. As pointed out earlier, this effect can never be compensated for by the slower decrease for large $|\phi|$. It is, however, possible to have negative solitary waves with two-temperature Maxwellian or kappa distributions (Baluku et al. 2010; Baluku and Hellberg 2012). These share the qualitative properties of the Cairns distribution that allow such structures, a comparatively large negative value of $F^{\prime}\left(v^{2}\right)$ near the origin, producing the excess of ions over electrons around $\phi=0$ needed to make $\Phi^{\prime \prime}(0)<0$. This is followed by a flatter shoulder which slows the rate of decrease of electron density as $\phi$ becomes more negative and for suitable parameters gives an excess of ions over electrons enough to produce a negative solitary wave.

\section{Conclusions}

In this short note, the behaviour of what has become known as the Cairns distribution in giving rise to both positive and negative ion sound solitons is examined. It has been noted previously that this property is not shared by other distributions such as the kappa distribution and the reason has been shown to be that such a distribution, which mainly differs from the Maxwellian in the nature of the high energy tail does not have a large effect on the behaviour of the electron density 
for negative potential. On the other hand, the flattening of the Cairns distribution around the origin and its almost flat shoulder means that the electron density goes down comparatively slowly for negative values of the potential. This results in a range where the electron density exceeds the ion density, a necessary condition for a negative solitary wave. Similar properties are shared by two-temperature Maxwellian and kappa distributions.

\section{Acknowledgements}

I would like to dedicate this paper to the memory of Padma Shukla, a good friend for many years.

\section{References}

Cairns, R. A., Mamun, A. A., Bingham, R., Boström, R., Dendy, R. O., Nairn, C. M. C. and Shukla, P. K. 1995 Geophys. Res. Lett. 22, 2709.

Chuang, S-H. and Lau, L. N. 2009 Phys. Plasmas 16, 022901.

Baluku, T. K. and Hellberg, M. A. 2012 Phys. Plasmas 19, 012106.

Baluku, T. K., Hellberg, M. A. and Verheest, F. 2010 Europhys. Lett. 91, 15001.

Sagdeev, R. 1966 In: Reviews of Plasma Physics, Vol. 4 (ed. M. A. Leontovich). New York: Consultants Bureau.

Tsallis, C. 1988 J. Stat. Phys 52, 479.

Vasyliunas, V. M. 1968 J. Geophys. Res. 73, 2839. 